\title{
JOIN'T MEETING OF COMMISSIONS II, 12, 13, 28, 29 AND 36 FOR A DISCUSSION ON EMISSION LINES
}

On Saturday morning, August 6, a joint meeting was held of Commissions II, I2, 13, 28, 29 and 36 for a discussion of Emission Lines. The meeting was presided over by Sir Arthur Eddington; Dr Swings acted as recorder. A general introduction was given by Prof. Russell, while separate phases of the subject were introduced by Drs Zanstra, Menzel and Merrill. Dr Lyot finally gave a review of his work on the photography of the corona and prominences without eclipse, and showed an impressive moving picture of the motions of prominences. The interest in this film was so great that Dr Lyot was asked, and consented, to present it a second time on Tuesday, August 9, on which occasion also a number of recent eclipse films were shown.

The various introductory talks will be given below in full.

The morning's session was almost entirely filled by these talks; the discussion was limited to a few remarks:

Dr MENZEL said that he did not agree with the commonly adopted idea that the forbidden transitions are strong in the nebulae because of the very low density of the nebular matter. Actually, the intensity of a spectral line is

with

$$
\text { Intensity }=\mathfrak{N} p h \nu,
$$

$\mathfrak{N}=$ number of atoms in the excited state considered;

$p=$ probability of the transition;

$\nu=$ frequency of the emitted radiation.

The factor $\mathfrak{N}$ will be larger in case of frequent collisions, i.e. for rather high densities.

Thus: the chief reason for the great intensity of the forbidden lines in the nebulae is the large amount of matter in those nebulae.

Prof. Stratron remarked that Dr Lyot has not observed several coronal lines which the Japanese astronomers thought they found in their coronal spectra of the 1936 eclipse, and Dr Lyot is sceptical about these lines. It is possible that the discrepancy results from the fact that the Japanese astronomers have observed their lines-especially the lines coinciding with the forbidden lines of NII-in the regions of high excitation of which Dr Menzel has spoken.

Dr Menzel is likewise sceptical, just as Dr Lyot. He has not observed the lines published by the Japanese astronomers, whereas they ought to be visible on his plates. Moreover, the chromospheric lines with which they might eventually be identified do not sufficiently agree in wave-length with the coronal lines given by the Japanese. Finally, the Japanese astronomers thought that they found new evidence, with a prism spectrograph, of the line $\lambda 6776$, which had formerly been observed with a grating, and which is now known to be the second order of the strong line $\lambda 3388$.

Prof. RUSSELL drew attention to the forthcoming publication, in I939, of a table, edited by Prof. Harrison, of the Massachusetts Institute of Technology, containing about Ioo,000 atomic lines. This volume, of considerable utility to all spectroscopists, will be sold at a moderate price; the American Government does not share in the cost of publication, but it has given subsidies for the preparation of the table.

$$
426
$$




\title{
EMISSION LINES IN ASTRONOMICAL SPECTRA
}

\author{
By H. N. RusSELl
}

A general survey of such varied phenomena as are included in the appearance of emission lines in the heavens must be either voluminous or somewhat sketchy. The latter course is indicated here.

I. The presence of emission lines (even of unknown origin) indicates certain important facts about the conditions under which they originate.

I. Emission lines appear only under conditions widely departing from thermodynamic equilibrium. "Absorption" lines, indeed, depend also upon such departures - there would be none in a Hohlraum-but emission lines, in general, demand more extensive deviations.

2. Such lines must originate in gas of very low, or at least fairly low density, where collisions are rare.

3. It follows that the energy of these radiations is not autogenous (originating within the emitting gas) but is fed into this gas in some other form and transformed by the emitting atoms.

4. In many cases it is evident (and in all cases probable) that they arise in an envelope of low density external to the original source of the energy, sometimes adjacent to it and sometimes very remote.

Study of the individual lines shows that

5. Almost all of them - with the important exception of the coronal lines-have been securely identified with radiations of known physical origin.

6. These are of very varied types-atomic (as in stars and nebulae) and molecular (as in comets and the aurora); ultimate (such as the D lines, $\mathrm{H}$ and $\mathrm{K}$, and the bands of $\mathrm{CN}, \mathrm{CH}$, etc.) and subordinate (Balmer series, helium); permitted (as in the flash-spectrum and most others) and forbidden (as in nebulae and the aurora).

7. Many observed emission lines are so wide that they have till recently been called "bands" (as in novae and Wolf-Rayet stars); but there is very strong evidence that this broadening is due to the Doppler effect. When the latter is absent (as in the aurora) the lines are very sharp. The moderate widening of the lines in planetary nebulae, etc., is probably due to radial motions. Two cases are known in which the widening is probably intrinsic: (a) $\mathrm{H} \alpha, \mathrm{H}, \mathrm{K}$, and some other lines in the flashlayer, where abundance of the emitting atoms is probably responsible, and $(b)$ the coronal lines, whose great width has been attributed tentatively to a very short life of the (unknown) emitting energy states.

8. Emission lines (in the observable region between 8000 and $3000 \mathrm{~A}$.) are very rare in the spectra of stars in which the maximum energy of the continuous background falls in this region (Classes $\mathrm{A}$ to $\mathrm{K}$ ) but are much more common when this maximum is in the infra-red (Class $M$ ) in the ultra-violet $(O, B)$.

II. Emission lines can obviously best be studied in cases where the emitting gas can be observed separately from the exciting source. We then find evidence of excitation of at least four different kinds: resonance, fluorescence, collisions and recombination.

(a) The classical example of resonance is the flash-spectrum which is doubtless excited thus - the circumstances explaining the presence of subordinate lines of all sorts and molecular bands. Comets' tails afford a very beautiful example, as has been emphasized by the recent work of Dufay and others. All the many bands which have been identified have the normal state of the molecule as their lower 
level. The dawn-flash appears to be another example. The high ionization in this and in comets cannot be due to this resonance.

(b) Fluorescence is beautifully exhibited in the gaseous nebulae-best of all by Bowen's explanation of the presence of a few selected lines of OIII and NIII produced as a result of the absorption of resonance radiation of one atom in an almost coincident resonance line of another and a second repetition of the process. This is a most remarkable observable result of "chance" agreements in wave-length and has been described as "the Odyssey of a quantum". Much of the radiation of the subordinate lines of hydrogen and helium may arise from stimulation by lines of the principal series. These resonance lines must be very strong.

(c) Excitation by impact of electrons or other charged particles is the only explanation of the light of the aurora. Bowen gives good reasons to suppose that collisions bear an important part in stimulating the forbidden lines in nebulae.

(d) Recombination of ions and electrons may have a considerable share in producing bright lines of the Balmer series (and probably others) in various sources. Its effects are not easy to disentangle from the others.

(e) Still another process-dissociation of molecules into excited atoms-has been suggested to explain certain emissions in long-period variables such as $\lambda 457$ I of $\mathrm{Mg}$.

Though the proximate source of the energy of excitation is thus varied, its ultimate source appears to be almost always the same-short-wave radiations emitted from a hot or very hot body - the one important exception being in the case of the aurora.

Bowen has shown in beautiful detail how the gradual absorption of this radiation (beginning with the shorter waves) accounts for the concentric stratification of the observed radiations in the planetary nebulae (and probably also in the Orion nebula, etc.).

Zanstra has applied similar principles to obtain minimum effective temperatures for the central stars. The results are high, though not incredibly so. The numerical results should be taken with caution, for they depend on the assumption of blackbody radiation. This now seems doubtful, for there is strong and increasing evidence that it is not true for the Sun and some other "cool" stars. The strong excitation of $\mathrm{HeI}$ in the chromosphere and the presence of HeII are thus explicable (Menzel), also the high ionization in the dawn-flash and in comets (Saha) and the excitation of gaseous nebulae by $\alpha$ Cygni and $\gamma$ Cygni (Hubble, Struve).

III. Emission lines in stars which appear telescopically single suggest the existence of envelopes too close to be directly resolved. There are several distinct classes of such objects.

(a) Hot stars with emission lines. Here we may set up an apparently almost continuous sequence from planetary nebulae and novae, where the existence of the emitting envelope is certain, through Wolf-Rayet stars where it appears extremely probable, to ordinary Be stars. (It is not suggested that there is a continuous sequence of physical processes involved in the production of the envelopes!)

In normal Be stars only a few lines are bright-often only those of hydrogen, sometimes also He, FeII, etc. A noteworthy property of these spectra is their variability. The emission often vanishes, or appears, or shows fluctuations with intervals of a number of years, but not exactly periodic. Evidently the condition which gives rise to the emission depends on a rather delicate balance of conditions which is easily disturbed. For these stars it is always permissible to assume an extensive envelope of low density and plenty of ultra-violet light. 
The $\mathrm{H}_{\alpha}$ line is always the brightest and the ultra-violet hydrogen lines are almost always dark. This is a natural consequence of the gradient of intensity, down toward the violet in emission and up in the continuous background. The lines are often "double" and the components are not infrequently unequal, with fluctuating alternations of intensity. Struve's hypothesis of a rotating envelope, varying in extent from time to time, should explain much of this, but a good deal remains puzzling. Study of spectroscopic and especially of eclipsing binaries which show emission lines should be instructive; but the best known of these is the notorious enigma Beta Lyrae.

(b) There are very few stars of classes $\mathrm{A}$ to $\mathrm{K}$ which show emission lines. Those which do are often variables of peculiar types. Some long-period Cepheids (W Virginis) and eclipsing variables (RS Ophiuchi) show emission lines. These stars would repay a thorough study but are unfortunately faint.

Cool stars with emission lines fall into two very different groups.

(c) In the long-period variables we find a complicated set of bright lines varying cyclically with the period. The primary emission appears to be that of the Balmer series, and perhaps some other resonance lines. Thackeray, assuming emissions to exist in the inaccessible ultra-violet, explains many of the emission lines in the violet, including the peculiar selection of certain members only of a multiplet-by fluorescence. The weakness of the bright $\mathrm{H} \epsilon$ indicates strongly that the hydrogen emission takes place at a level below much of the CaII absorption. There is no doubt that these stars have enormous envelopes of low density. If radiation in the far ultra-violet escapes from the interior up to the level where the temperature is comparable to that of the Sun's surface, hydrogen emission may be stimulated, and if the outer layers are full of molecules such as $\mathrm{N}_{2}$ which absorb very strongly in the ultra-violet, the main emissions might lie below a good deal of the CaII. The details in these variables are complicated and are far from being explained.

(d) A number of dwarfs of class $M$ show bright hydrogen lines. This is one of the most puzzling of emission phenomena. These stars-Castor $C$ is one of them-are normal dwarfs with surface gravity so high and radiation pressure so low that extensive envelopes seem to be out of the question. Strong radiation in the remote ultra-violet might provide the excitation, but Wildt has pointed out (in conversation) that there should be enough molecules such as $\mathrm{H}_{2}, \mathrm{~N}_{2}$, etc., in the atmospheres of these bodies to obstruct the short waves completely.

In conclusion it may be noted that there is a decided tendency for variable stars to show bright lines at minimum (e.g. R Coronae Borealis, Merrill's "iron star", SS Cygni). As neither the cause of the variation nor of the emission is known the problem must be deferred.

\section{EMISSION LINES IN NEBULAE AND NOVAE}

\section{By H. ZaNsTra}

When one is faced with the problem of giving a talk of about twenty minutes on the subject of emission lines in nebulae and novae, one may be forgiven for imposing some drastic limitations. I shall limit myself, therefore, mainly to a discussion of the processes of emission, and, for the novae, to the well-studied recent case of Nova Herculis. For the sake of brevity, I shall have to be very unjust to those workers who have laid the foundations.*

* In the case of nebulae in particular Hubble and the Lick observers, and for the novae Halm, Beals, Menzel, Mrs Payne Gaposchkin and others. 
Emission lines in nebulae. A gaseous nebula may be conceived of as a cloud of gas, which is excited to luminosity by a star of high temperature, say between 18,000 and $100,000^{\circ}$. The dimensions of the cloud are generally quite large as compared with the star's radius, so that the exciting radiation is very dilute. Under those conditions, the following three mechanisms have proved useful for studying the observed luminosity of the nebulae.

In the first place, the atoms of the nebula, which are very predominantly in the ground state, may be ionized by the absorption of the star's continuous spectrum in the far ultra-violet beyond the ionization limit of the atom. Subsequent recombination, however, must take place on all levels, after which the electron goes down by cascade jumps, and this gives the emission of a number of bright-line spectra and continuous spectra at their heads, e.g. the Balmer series of hydrogen

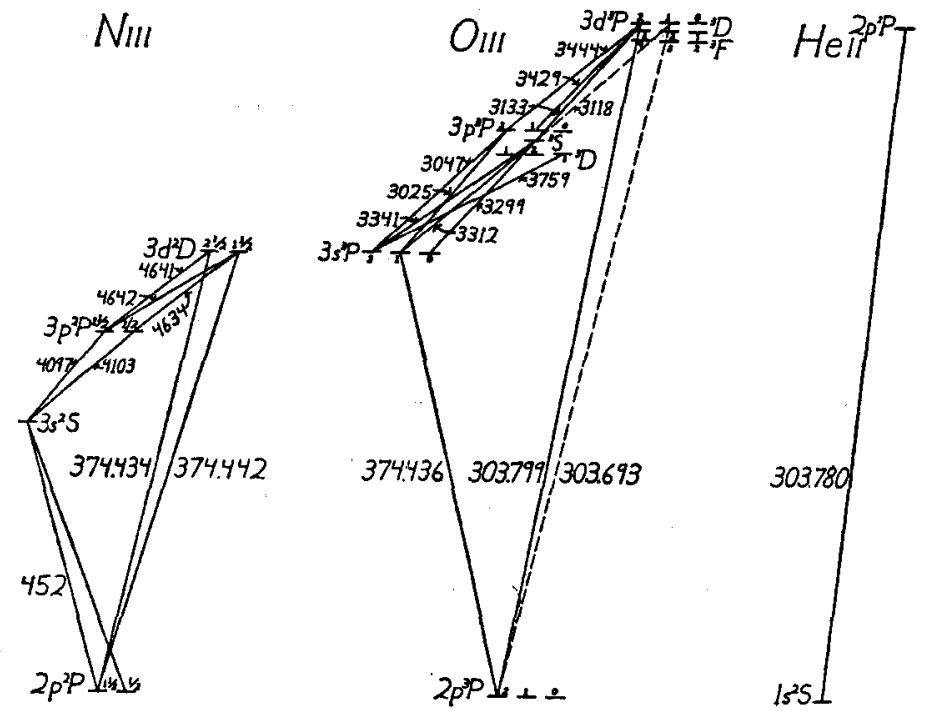

Fig. r. Excitation of the permitted lines of OIII and NIII by fluorescence according to Bowen. (From I. S. Bowen, $A p . J .81,1$, 1935, Fig. 1.)

and the $\mathrm{Ba}_{c}$ spectrum. The series of lines corresponding to jumps from a higher to the very lowest level is rapidly converted into its first line: Lyman $\alpha(L \alpha)$ in the case of hydrogen and ionized helium.

Before the photo-electrons recombine, they can give up their energy by exciting lines of low excitation, which, in the case of nebulae, all happen to originate from metastable states. Such forbidden lines, the so-called Nebulium lines, are therefore all excited by this second mechanism of electron impact, first suggested by Bowen. Examples are the lines of $[\mathrm{OII}],[\mathrm{OIII}],[\mathrm{NIII}]$, etc., the square bracket indicating that the line is forbidden.

But there is yet a third way, likewise due to Bowen.* The line $L \alpha$ of ionized helium, which is emitted according to the first mechanism, has a wave-length almost exactly equal to that of a double absorption line of the ion $\mathrm{O}^{++}$, so it can excite some very definite lines of the spectrum OIII by fluorescence (see Fig. r). This story repeats itself once more for the spectrum NIII, as is shown in Fig. I.

* I. S. Bowen, $A p$. J. 81, I, I 935 . 
The observed fact is now that all permitted lines of OIII and NIII in the nebulae are among those indicated in Fig. I, whereas in the laboratory spectrum also many other strong lines occur. Needless to say that they only occur in the nebulae when the spectrum of $\mathrm{He}^{+}$is present giving the required $\mathrm{L} \alpha$.

If we return to the first two mechanisms, it is clear that a photometry of star spectrum and nebular spectrum should yield the temperature of the star, if this is assumed to be a black body. The fact is that the stellar temperature required to produce the hydrogen recombination is close to that required for the excitation of the nebulium lines by electron impact.* The stellar temperature required to produce the observed $\mathrm{He}^{+}$spectrum by recombination is, however, a good deal higher, as Berman showed. $\dagger$ This probably means that beyond the ionization limit of $\mathrm{He}^{+}$the star is richer in ultra-violet than a black body.

The outstanding difficulty at present is, that the observed ratio of $\mathrm{Ba}_{c} / \mathrm{Ba}$ in nebulae as derived by Pagef is an enormous factor smaller than would correspond

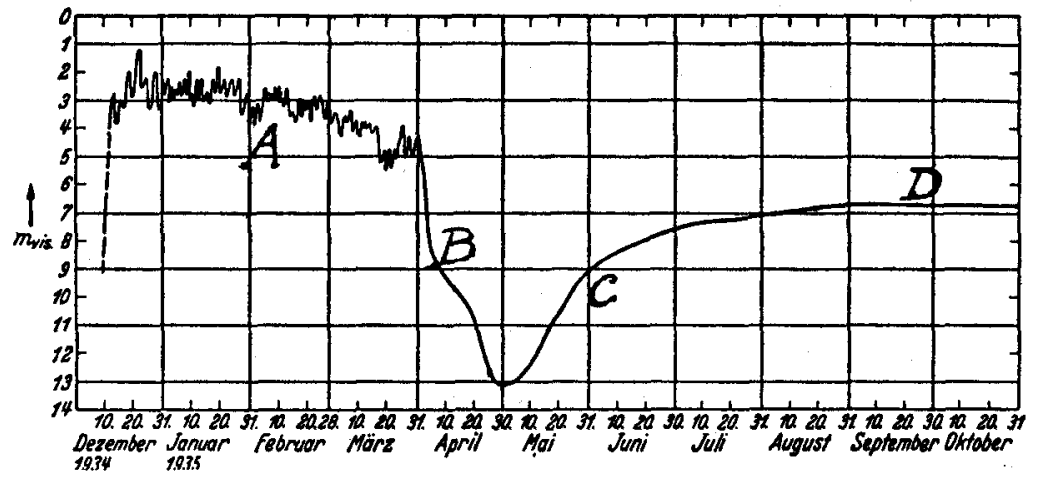

Fig. 2. The light curve of Nova Herculis, 1934. (From W. Grotrian, Z. f. Ap. I3, 215, 1937, Fig. I with the stages A, B, C and D marked.)

to its theoretical ratio according to Kramers's formula, as was shown by Cillié.ई The laboratory spectrum observed by Page under conditions favouring pure recombination shows the same discrepancy.

Emission lines in Nova Herculis 1934. A normal nova generally rises to its maximum in a few days, and then fades rapidly, going down by something like five magnitudes in a month. Compared with such normal behaviour, the light curve of Nova Herculis shown in Fig. 2 is exceptional in more than one way. Before the outburst it was of the 15 th magnitude. When discovered by Prentice on December I2, I934, it probably was rapidly rising, then it rose more slowly to the not very pronounced maximum of December 22. From here on one may distinguish the following portions: $\Uparrow$

A. December 22, I934 to April I, I935, during which it went down but slowly, with oscillations.

* H. Zanstra, Publ. D.A.O. 4, 209, 1931; Z.f. Ap. 2, 1, 193r.

$\dagger$ L. Berman, Lick Obs. Bull. No. 430, 1930.

$\ddagger$ T. L. Page, M.N. 96, 604, 1936; Comm. Univ. Obs. Oxford, No. 7 .

\& G. G. Cillie, M.N. 96, 77I, 1936.

II T. L. Page, Nature, 141, $1137,1938$.

Tा The only similar case is that of Nova Aurigae 1891 , as was e.g. pointed out by Beer (M.N. 95, 538, 1935), who refers to the light curve of Campbell (Harv. Ann. 84, 153, 1920, Fig. 1). 
B. A rather steep drop between April I and April 30, 1935 .

C. After this very pronounced minimum a rather smooth increase, gradually merging into

D. A nearly stationary state (by the end of 1936 the star was $7^{\mathrm{m}} \cdot 4$ ).

It is this very abnormality which had made Nova Herculis most interesting from a theoretical point of view, since, as will be seen, the slowly decreasing portion A serves for testing Kosirev* and Chandrasekhar's $\dagger$ theory of extended photospheres, and the non-stationary portion $\mathrm{C}$ for testing the theory that the bright-line spectrum is due to recombination, as in the nebulae.

As in the case of other novae, there must have been a succession of explosions in which matter was thrown off by the star, since the absorption and emission lines show a complicated structure. t The two main masses thrown off presumably in opposite directions became separately visible on July I, I935, when Kuiper observed a doubling of the visual image. Combining the observed separation $\mathrm{o}^{\prime \prime} \cdot 2 \mathrm{I}$ with the observed radial velocity of 300 to $400 \mathrm{~km}$./sec., Russell $\S$ finds a distance of about 400 parsecs, agreeing with Williams's determination $\|$ from the intensity of interstellar calcium and with the mean of other values.

But in addition to this series of explosions, the portion $\mathrm{A}$ of the light curve requires the assumption of a more or less constant streaming out of matter up to April I, I935. Such a stationary stream of matter emerging from the star evenly in all directions will have the effect of enormously extending the star's atmosphere. Assuming local thermodynamic equilibrium at each point of the stream, one can assign a temperature $T$ at any radius $r$. Since the density of matter is not high, the radius varies considerably with the optical depth, which results in a change of temperature with optical depth much more considerable than for the plane case. This means that the radiation from such an extended photosphere shows large deviations from the black body distribution. Calculation shows that it is much richer in the ultra-violet.

The main assumptions of Kosirev and Chandrasekhar's theory are in short: (I) a stationary stream, (2) local thermodynamic equilibrium, (3) a mass absorption coefficient independent of the wave-length and proportional to $r^{-\frac{1}{2}}$ or, what is the same, $P T^{-5}, P$ being the electron pressure. T The approximate physical justification of the latter assumption was discussed by Whipple and Mrs Payne Gaposchkin.**

If one supposes that the density of matter varies directly as the total outgoing energy, the situation is entirely determined by the latter, and one may predict, apart from a constant, the relation between colour temperature $T_{0}$ and stellar magnitude $m$. Whipple and Mrs Payne Gaposchkin find that the observations of $T_{c}$ and $m$ for the portion A fit this relation.

Cecchini and Gratton $\uparrow \uparrow$ have measured, in stage $\mathrm{A}$, the intensities of the hydrogen emission lines expressed in terms of the adjoining continuous spectrum. Assuming excitation by photo-ionization and recombination in the outlying gases, one may,

* N. A. Kosirev, M.N. 94, 430, 1934 .

† S. Chandrasekhar, M.N. 94, 444, r934; Proc. Camb. Phil. Soc. 31, 39o, r935.

$\ddagger$ Cf. D. B. McLaughlin, Publ. Am. Astr. Soc. 8, 145, 1935; Publ. Univ. of Mich. Obs. 6, 107, 1937; A. Beer, M.N. 96,236 , I936.

$\S$ H. N. Russell, Publ. A.S.P. 48, 32, 1936.

II E. G. Williams, M.N. 95, 573, x 935 .

T Chandrasekhar discusses also some other values of the exponent.

** F. L. Whipple and C. Payne Gaposchkin, Harv. Circ. No. 412, 1936.

†† G. Cecchini and L. Gratton, Mem. d. classe di sc. phys. math. e nat. Reale Accademia d'Italia, 8, 654,1937 . 
from these intensities, derive a stellar temperature, as in the case of the nebulae. Now this photo-electric temperature is found to be around $23,000^{\circ}$, whereas the colour temperatures are around II,000. Cecchini and Gratton show that an extended photosphere would behave approximately in this manner: if, for the ionization of hydrogen, it acts like a black body of $23,000^{\circ}$, it behaves in the visual region like a black body of about $9000^{\circ}$. So the theory of the extended photosphere generated by the stream is confirmed.

We now come to the explanation of the sudden drop $B$ and subsequent rise $C$ by Grotrian.* Grotrian assumes that about the first of April the stream has given out. As the outside matter dilutes, the extended photosphere becomes less and less important, and this explains the drop B. About April 30 the extended photosphere has disappeared and the comparatively faint star at the centre is unveiled. This new star, though faint, must be very hot. From the intensity of bright lines in the final stage $\mathrm{D}$, Oehler $\dagger$ determined its temperature to about $70,000^{\circ}, \ddagger$ and this hot star is now suddenly "switched on".

But the effect on the outside gases is not sudden. At first the number of recombinations is much less than the number of ionizations, and it only gradually catches up with it. So Grotrian gives for the recombination luminosity $I$ the formula

$$
\frac{I}{I_{\infty}}=\left(\frac{I-e^{-2 \sqrt{a \bar{N}} \cdot t}}{I+e^{-2 \sqrt{a \bar{N}} \cdot t}}\right)^{2}, \quad \alpha=\frac{\alpha^{\prime}}{V},
$$

where $\alpha^{\prime}$ is the recombination coefficient per $\mathrm{cm} .{ }^{3}, V$ the volume of the envelope and $N$ the total number of ionizations in the whole gaseous envelope per second. (N.B. not per $\mathrm{cm} .^{3}$ )

For an electron temperature of $10,000^{\circ}$ one has for the recombination coefficient per $\mathrm{cm} .{ }^{3} \alpha^{\prime}=2 \cdot 3 \mathrm{I} \times \mathrm{IO}^{-13}$. The observed average expansion velocity of $450 \mathrm{~km} . / \mathrm{sec}$. for 200 days leads to the volume of the gases $V=2 \cdot I 4 \times 10^{45} \mathrm{~cm}^{3}$. One gets then an almost perfect fit with the light curve $C$, if one adopts for the total number of ionizations per second $N$ the value

$$
N=2.4 \times 10^{44} \mathrm{sec}^{-1} \text {. }
$$

On the other hand, if the visual magnitude of the star is estimated as $I 5^{\mathrm{m} \cdot I}$ (viz. 2 magnitudes fainter than the minimum), at a distance of $4 \mathrm{r} 5$ parsecs, its temperature of $70,000^{\circ}$ leads to a radius which is $\frac{1}{40}$ of that of the Sun. Such a star emits in total a number of ultra-violet quanta beyond the head of the Lyman series

$$
N=\operatorname{II} \times 10^{44} \mathrm{sec}^{-1} \text {, }
$$

which gives rise to an equal number of ionizations per second for the hydrogen in the envelope.

So there is agreement as regards order of magnitude. $\S$

The outcome is very favourable for the recombination mechanism as causing the luminosity. It also gives a great deal of confidence that the mechanism for

* W. Grotrian, $Z$. $f . A p$. 13, 215, 1937; Z. f. Techn. Phys. 18, 146, 1937.

† H. Oehler, $Z$. f. $A p$. 12, 281, 1936.

$\ddagger$ Oehler obtained this from the ratio $\mathrm{He}^{+} / \mathrm{H}$ according to Ambartsumian. From the rise of $13^{\mathrm{m}} \cdot \mathrm{I}$ to $6^{\mathrm{m}} \cdot 7$ one finds that the difference in visual magnitude of star and "nebula"

$$
m_{*}-m_{n} \geqslant 6 \cdot 4 \text {, }
$$

or $\geqslant 5.9$, if the photographic magnitude is used for the star, colour index -0.5. Then $Z . f . A p .2$, 26 , I93I, Table 6 , yields $T \geqslant 70,000^{\circ}$. This confirms the high temperature.

$\S$ As Grotrian remarks, it would be easy to force an agreement, e.g. by assuming an electron temperature of $50,000^{\circ}$. 
nebulae is correct, where we have likewise a cloud of gas excited by a star of dimensions small compared with the cloud.

We shall now very briefly consider the forbidden lines. For atoms or ions with 2,3 or 4 -electrons, the excited states 2 and 3 are metastable, and there are good grounds for believing that the second state has the longest life* and is thus, more easily than the third, destroyed by collisions of the second kind. In the earth's upper atmosphere e.g. the density, though small, is yet sufficiently high to destroy very appreciably state 2 of OI $\dagger$ and so we have in the aurora predominantly the transition 3, 2, which Menzel and Mrs Payne Gaposchkin $\ddagger$ have termed the auroral transition.

In earlier stages of novae when the density is yet high enough state 2 is destroyed, so that the auroral transitions appear later than the nebular transitions, and become gradually more important as time goes on. $\$$ For Nova Herculis in stage D, Dufay and Bloch $\|$ find that the intensity ratio of "nebular" [OIII] to "auroral" [OIII] increases with time, whereas also the intensity of nebular [NIII] increases, just as could be expected. T

The forbidden line of $\mathrm{Fe}^{+}, \lambda 4287$, appears during the drop $\mathrm{B}$ of the light curve and is absent during state $A$. Grotrian suggests the following explanation. The line originates from the metastable level 2, but 3 is not metastable. During state A, when the continuous spectrum is very strong, all atoms may be lifted from this metastable level 2 to the instable level 3 by absorption of the permitted visual line $\lambda 4923$ from the continuous spectrum, and then can pass into the ground state by the transition 3, I. So here the metastable level would be destroyed by absorption of radiation rather than by collisions of the second kind.

We have seen, in the case of the nebulae, that certain permitted OIII and NIII lines may be excited by fluorescence due to absorption of the line $L \propto$ of $\mathrm{HeII}$. Dufay and Bloch show that also in Nova Herculis in stage D those lines are the strongest which can be excited by this mechanism. So also Bowen's mechanism of fluorescence was tested in the case of Nova Herculis.

Altogether Nova Herculis, in the hands of many able investigators, has proved a most valuable object for testing theories: the theory of the extended photosphere and the excitation of the hydrogen lines, the excitation and extinction of forbidden lines, and the excitation of permitted lines by fluorescence. Also it seems to have provided a large-scale experiment in recombination, where the ionizing source, nothing less than a star, was switched on, and thus has strengthened the somewhat shaken confidence for this mechanism in the case of nebulae. Its course of evolution was a rather exceptional one, but it seems that its very abnormality has made Nova Herculis a most interesting object of study to the delight of both observer and theorist.

* The transition 2, r between a singlet and a triplet level (intercombination line) is less probable than the transition 3,2 between two singlet levels.

† I. S. Bowen, Phys. Rev. 36, 600, 1930.

$\ddagger$ D. H. Menzel and C. H. Payne, Proc. Nat. Ac. Sc. 19, 641, 1933.

\$ Menzel and Payne, loc. cit. The predominance of auroral transitions in novae as compared with nebular transitions in nebulae was first explained in this manner by Grotrian for [OIII] and [OI], $Z$. $f$. Phys. 60, 302, 1930; $Z . f . A p .2,78,193$ I.

II J. Dufay and M. Bloch, $Z$. f. Ap. 13, 36, 1937 .

If Since the time was rather limited, the following paragraph discussing the other bright lines was not read at the Stockholm meeting. 


\title{
CHROMOSPHERE AND CORONA
}

\author{
By Donald H. Menzel
}

Among the unsolved problems of astrophysics those related to the solar atmosphere are probably the most important. The sun is the only star where the surface structure can be studied in detail. The sun is a laboratory, which may be used for developing, revising and checking theories of stellar atmospheres. Thus far, unfortunately, most of the emphasis has been on developing and revising. The sun perversely refuses to check most of our theories. Defects in our hypotheses are not so evident as we study the fixed stars, but the sun is a trifle too close for astrophysical comfort.

Among the anomalies that worry us are the great strengths of the hydrogen and the infra-red helium lines in the Fraunhofer spectrum. Stark effect almost accounts for the former but the helium discrepancy cannot possibly be so explained. I mention these lines of high excitation because their occurrence in absorption is but an indication of the much greater discrepancy in the chromosphere, where, in addition to excitation, we find ourselves faced with the allied problems of levitation and turbulent activity. These anomalous phenomena are not improbably a universal characteristic of stellar atmospheres and may exert an appreciable effect on the intensities of spectral lines requiring high excitation. The strength both in emission and absorption of hydrogen lines in late-type stars, and the supposed turbulence in the atmospheres of certain super-giant stars may arise from causes not dissimilar to those operating in the solar chromosphere. Without an understanding of the processes involved we may scarcely expect to achieve a complete quantitative explanation of stellar spectra.

We should all prefer to approach the problem as did our present chairman (A. S. Eddington) in studying the internal constitution of the stars. With pencil and paper as tools and with the laws of physics to guide us, we should like to deduce the stars from core to corona. Which are the more complicated, stellar atmospheres or stellar interiors, I am incompetent to judge. But those of us whose chief concern is the outer atmospheric shell, consisting of only $10^{-12}$ or $10^{-13}$ part of the stellar mass, find this shell producing difficulties altogether out of proportion to its mass. The subjective method of approach has failed completely and we are forced to rely on a somewhat less elegant but definitely safer method. We shall start with the observational data and see what they can tell us about the physical conditions obtaining in the atmosphere. Guided by the data we may eventually hope to discover where our previous theories have gone astray.

Observation, then, takes priority. Thus far, in chromospheric spectro-analysis, eclipses have furnished the major portion of the material. The studies of Lyot, at Meudon and at the Pic du Midi, represent the most important developments in coronal research during recent years. We have, at last, a number of accurately determined coronal wave-lengths. Lyot has, in addition, added five new and important lines, most of them in the infra-red. Finally, by photographing the corona directly, without eclipse, he obtains frequent records of coronal phenomena from which we may hope, in the future, to derive the spatial structure of the corona, determine its internal motions and fix its relationship to sunspots, chromosphere and faculae. Lyot and his observations will presently speak for themselves on this same programme. But I take this opportunity to pay tribute to the skill 
of an astronomer who has solved the problem that many scientists have declared insoluble.

With the development of these newer instrumental techniques and with the wealth of accumulated eclipse data, those of us who in the future may contemplate eclipse observation have an added responsibility. We must remember the neglected, perhaps not so spectacular, but none the less important fields of solar research, from which eclipse expeditions may withdraw much-needed financial support. Future observational eclipse programmes require more careful planning with the object of supplementing rather than replacing non-eclipse research. Coronal spectra in the far ultra-violet, where atmospheric scattering leads to difficulties with Lyot's equipment, are particularly needed. Specialized interferometric and polarization studies of emission lines, both coronal and chromospheric, are also particularly to be desired. Careful, quantitative photometric standardization of every spectrum is, of course, an absolute necessity.

At the present time I am able to give a brief summary of some results obtained at the 1936 eclipse by the Harvard-M.I.T. expedition to Ak-Bulak, U.S.S.R. In studying the flash and coronal spectra we have obtained, thus far, approximately two kilometres of micro-densitometer tracings. About half of these have been reduced to give absolute intensities. We expect eventually to publish a fairly complete table of intensities of lines at different chromospheric levels and also of lines of several different prominences.

The observations now available clearly establish the variability of the chromospheric spectrum. It varies not only with time but from point to point around the solar limb. Both the intensities and intensity gradients are subject to wide variations. I can illustrate best how marked the differences are by saying that the spectra of two neighbouring chromospheric regions may be as unlike as the spectra of $\mathrm{F}$ - and $\mathrm{K}$-type stars.

I had noticed minor variations on spectra obtained prior to I932, but the spectra I secured in that year showed the anomaly particularly clearly. The chromosphere was fairly uniform around the solar disk except in one small arc, about 50,000 $\mathrm{km}$. in length, where distinct evidence of high excitation was found. In this region the lines of hydrogen, neutral and ionized helium, ionized silicon, etc., were greatly enhanced. Many lines of low excitation, including most band spectra, completely vanished.

In studying this interesting region, Cillie and I found what appears to be the first definite physical relationship between corona and chromosphere. Above the area of high excitation, the coronal continuum was very marked and the coronal lines strong. 5303, in particular, and other lines apparently associated with 5303 , scarcely showed at any other point around the disk. Except at this location, 6374, which appeared to be clearly ring-form, exceeded 5303 in intensity.

On this perhaps slender evidence, Cillie and I ventured to suggest that the correlation between chromospheric and coronal excitation was general. The r936 observations appear to have confirmed the conclusion. In I932 we had referred to the small arc as "highly excited" and to the rest of the chromosphere as "normal". The designation has proved to be somewhat unfortunate, for by these standards none of the 1936 chromosphere was normal ; all of it was at least "highly excited" and a large portion (especially that at first flash) was "super-highly excited". I have never seen the coronal lines, especially 5303, so strongly recorded, both on the relatively slow moving-film and on the more rapid jumping-film. In the latter exposures considerable coronal structure, suggestive of eruptive activity, was found. 
The corona was particularly brilliant over the sun-spot zones, where the helium lines were relatively much more intense than the metallic. In the equatorial zones, where the coronal lines were weak, the metallic lines were markedly enhanced. The appearance and behaviour of the spectral lines strongly suggest that the metals were undergoing second-stage ionization.

Our studies of the coronal spectrum are still far from complete. Each plate must be measured again and again, at different regions, to eliminate spurious lines and to check the wave-lengths. Intensities are also being measured, although the relative intensities of different lines vary so greatly around the disk that one has difficulty in deciding upon a standard. We have found most of the coronal lines recorded in the more conservative lists and have checked several that have been dropped from the more complete Lick list of I9r8. In addition we have detected a number of new lines, the strongest of which was $\lambda 7060$, discovered independently by Lyot. An

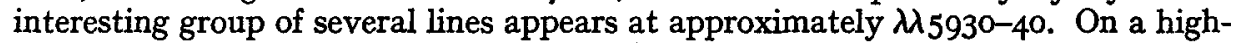
dispersion slit spectrogram $\lambda_{423}$ is seen to be a close doublet. $\lambda_{360 \mathrm{I}}$ is also probably double. Our classification of the stronger lines, by means of coronal form, agrees essentially with that of Mitchell. At least three and probably four distinct groups of coronal lines are found. Of particular significance is the fact that the coronal continuum appears to be always associated with $\lambda 5303$, and vice versa.

None of the many attempts to identify the coronal lines have been successful. It is possible, however, to draw a few general conclusions concerning the nature of the atoms involved in producing the lines. Several types of atoms, i.e. several elements or the same element in different ionization stages, are present. The ultimate solution must distinguish between the various line groups. The association between the helium and the coronal lines, in the spectrum of RS Ophiuchi as well as in that of the sun, clearly shows that high excitation stages are involved.

The inner coronal continuum is commonly attributed to scattering by free electrons. The chief evidence in favour of the hypothesis is the close correspondence of its spectral distribution with that of the sun. Also the calculated electron densities are not unreasonable. The values I have obtained from the 1936 measures are not far from those found in 1927 by Pannekoek and Minnaert. The one difficulty with the hypothesis lies in the complete absence of the $\mathrm{H}$ and $\mathrm{K}$ absorption lines. Our I936 spectra, taken with a dispersion of I A. per mm., show no indication of an intensity drop in the $\lambda 3900$ region; Grotrian has suggested that the lines are washed out by Doppler effect, but so complete a disappearance would require velocities of at least $10^{3} \mathrm{~km}$. per sec. Turbulent mass motion of this order of magnitude cannot be admitted, because the coronal lines would then be twenty or more Angstroms wide. The indicated minimum kinetic temperature is $30,000^{\circ}$ and it is probably much greater.

At the present time no more likely source than electron scattering is evident for the coronal continuum. I should mention that this interpretation of the observations fits in well with Rosseland's theory, which relies on collisions of fast electrons instead of on radiation for excitation and levitation of the chromosphere. I do not regard the question as entirely closed, however. We should still be alert for other atomic sources of continuous spectrum, sources that may simultaneously give rise to the associated $\lambda 5303$ group of lines. Processes involving double-electron excitation and auto-ionization should be particularly investigated. The original hope of explaining the coronal lines as the result of metastable atomic transitions, like those occurring in the gaseous nebulae, is fast dwindling. Edlén and Swings tell me that they have nearly completed the analyses of FeIII, the only remaining likely 
source of nebular-type lines. If this investigation fails to solve the corona problem, we shall have to take consolation, as does Dr Edlen, in the thought that the intriguing perplexities of the corona mystery have stimulated research of great physical and astrophysical value. We shall suffer indirectly when the stimulus is removed.

There are many problems related to the chromosphere which lack of time as well as lack of knowledge forbid my discussing more fully. Of special interest is the question of motions of prominences and of chromospheric spikes. How do these objects suddenly acquire high tangential velocities at great heights? Why do these velocities show the sudden accelerations observed by Pettit? Are electric or magnetic fields in any way involved? The cinematograph studies at the McMathHulbert Observatory and at the Pic du Midi should assist materially in resolving the problems. Are the prominence-like formations of the corona related to ordinary prominences? The difference appears to be more than one of relative excitation; otherwise we should expect to find intermediate cases of prominences showing both the ordinary and coronal spectra.

I return again to the most outstanding problem: chromospheric excitation and levitation. The Saha theory went far to explain the relative strengthening of lines of metallic ions as an effect of lowered pressure. But, in the extreme case of $\lambda 4686$ of the HeII, the discrepancy left by the Saha theory is of the order of $10^{12}$, if we assume the solar radiation to have an effective temperature of $6000^{\circ}$. Temperatures of from $I 5,000^{\circ}$ to $25,000^{\circ}$ are indicated, but, if the excitation is by radiation, all that is physically required is a radiation appendage considerably in excess of the $6000^{\circ}$ value, far in the ultra-violet.

If our preconceptions, based on theoretical premises, had not pointed so definitely to the contrary, we should long ago have concluded from the observational data that the excitation temperature of the solar atmosphere increases outwards. It seems that we can no longer escape from this conclusion. There is no obvious source of external excitation, as exists for the terrestrial atmosphere, in the upper regions of which a somewhat similar temperature inversion occurs. Cosmic radiation can exert only a negligible excitation effect, although accumulation of charge by the sun might result in expulsion of ions by electrostatic action and thus partially explain coronal and chromospheric levitation. At the present state of knowledge, both of cosmic rays and of the solar magnetic field, further pursuit of this speculative suggestion would be unprofitable.

There are no obvious ways in which ultra-violet radiation can either escape from the solar interior or be produced in the atmospheric layers. And yet the observations clearly show that a large amount is leaving the sun. I can think of only one possible explanation, against which so many obvious objections can be raised, that I shall not attempt either to list these objections or to defend the hypothesis. I am inclined to the view that the turbulent character of the solar atmosphere is not a mere surface phenomenon. Bjerknes has outlined an attractive theory of sunspots, where extended deep-lying vortex tubes rise to the surface. Observation points to a relationship between sun-spot and prominence activity and I hold that the chief distinction between prominences and chromospheric spikes is only one of size. Prominences reaching to greatest heights are presumably rooted in regions of greatest excitation. The excitation might thus be carried to great heights and the inverse temperature gradient accounted for. The stratification tends to be vertical rather than horizontal. If the exit spouts of the prominences occupy only a small fraction of the solar disk, the Fraunhofer spectrum would not be greatly affected, 
except in high-excitation lines. The "peppered" appearance of hydrogen spectroheliograms compared with the "orange peel" appearance of $\mathrm{K}$ spectroheliograms is in accord with the picture I have drawn.

Whether or not these concluding suggestions can be built into a quantitative physical theory I cannot say. I should like to point out, however, that the diffculties and serious objections will begin in the second rather than in the first paragraph of the proposed paper. I have avoided the assumption which, often appearing in the first sentence, has spelled the death of many an otherwise attractive theory: "Consider the sun to be uniformly stratified in parallel layers perpendicular to the solar radius."

\section{EMISSION LINES IN STELLAR SPECTRA}

\section{By Paul W. Merrill}

Emission lines occur frequently in the spectra of stars near the extremes of the temperature sequence, very rarely in intermediate types, the transition at either end of the series being abrupt. Thus from $\mathrm{Bo}$ to $\mathrm{B}_{3}$, at least one star in ten has bright hydrogen lines, but at Ao only about one per thousand. The maximum relative frequency of hydrogen emission superposed upon a normal early-type absorption spectrum appears to occur at $\mathrm{B}_{2}$ rather than among the still hotter Bo or $\mathrm{O}_{9}-\mathrm{O}_{5}$ stars. In the very hottest objects the Wolf-Rayet type of emission prevails. Among the low-temperature giant stars, bright lines are exhibited by nearly all of types M6 and later, but by only a very small fraction of those of type Mo.

Since emission lines are often sensitive to slight changes in the physical conditions under which they originate, and when observed in integrated star light may indicate a lack of gravitational or thermodynamic equilibrium in the stellar atmosphere, it is not surprising that they occur frequently in the spectra of variable stars and are subject to changes which are often very pronounced. A good deal is already known concerning the typical behaviour of emission lines in the spectra of long-period variables, but the phenomena are very complex and demand much further study. Spectroscopic observations of variables of the R Coronae, SS Cygni, and other rare types have shown remarkably diverse bright-line phenomena. The scope of these observations should be extended and detailed intercomparison of apparently similar behaviour in various objects made whenever possible. Eclipsing variables with bright lines offer especially interesting problems. In this general connection it should be noted that hundreds of non-variable stars (i.e. stars not known to be variable) have bright lines, and that in some instances considerable spectral changes have occurred. $\mu$ Centauri and $\mathrm{HD}_{50138}$, one with slow, the other with rapid changes, may serve as examples. It would probably be well to make accurate photometric tests of all bright-line objects, giving special attention to those known to be binaries or to exhibit variations in spectra. For B- and A-type stars the catalogue in Mount Wilson Contribution No. 47 ${ }^{*}$ might be a useful guide.

The proportion of bright-line objects among $c$ stars and others of especially low density is sufficiently high to be of physical significance. Low stellar density is thus correlated both with stellar variability and with a tendency toward bright lines. On the other hand, a number of late-type dwarf stars have conspicuous emission lines of hydrogen and calcium. The companion to Castor is a well-known example.

$$
\text { * Ap. J. 78, 87, } 1933 .
$$


The chief groups of bright-line stars are surprisingly well defined and homogeneous. In both early- and late-type stars hydrogen emission apparently tends to be fairly intense, if present at all, although this conclusion may rest partly on the fact that in the present state of stellar spectroscopy little attention is likely to be paid to a bright-line spectrum unless at least one line is conspicuous. Nevertheless many weak emission lines, especially weak bright borders to dark lines, are known (particularly in $c$ stars) and doubtless many others remain undiscovered. In addition there must be other lines wholly dark whose structure is modified by incipient emission. These facts demand that especial caution be used in the interpretation of the profiles and equivalent widths of lines which frequently appear bright, even in instances where no emission is visible.

\section{Early-Type Stars}

The chief elements represented by bright lines in B-type spectra are hydrogen, helium, and ionized iron. The approximate numbers of stars known, including a few comparable stars of types $\mathrm{O}$ and $\mathrm{A}$, and a few nondescript objects, are:

$\begin{array}{ll}\mathrm{H} & 460 \\ \mathrm{FeII} & 35+ \\ \mathrm{He} & 24+\end{array}$

The FeII lines are often ill-defined and difficult to recognize; extensive observations with widened spectra on contrasty emulsions would probably disclose many more. Similar observations, especially of the $\mathrm{D}_{3}$ region, would probably show a number of additional stars with weak helium emission.

In early-type spectra the profiles of bright hydrogen lines regularly form the pattern Fig. Ia or some variation of it. In a given star, marked changes in the

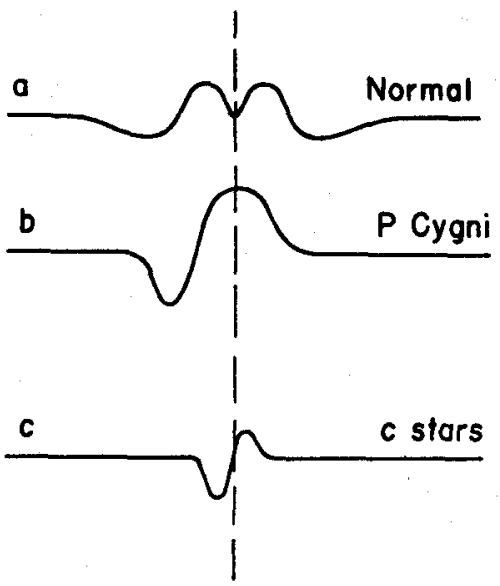

Fig. I. Typical profiles of bright hydrogen lines in early-type spectra.

relative intensities of the maxima and in their combined intensity are the rule. The changes are generally slow, a cycle often being measured in years. A few stars, like $\mathrm{P}$ Cygni, show structure of the nova type $\mathrm{Ib}$, while $\mathrm{H} \alpha$ in $\mathrm{c}$ stars usually resembles Ic.

The central minimum in Fig. I a, when sharply defined, may be called a "core". This feature is ascribed to high-level absorption; its appearance and extraordinary 
behaviour in many Be spectra (e.g. $\phi$ Persei, $\zeta$ Tauri, HD 31293, HD 142983) offer many interesting opportunities for special investigations. The phenomenon may be analogous to the well-known self-reversal of arc lines in the laboratory.

Bright lines in early-type spectra probably arise from an unusually extensive glowing atmosphere. A gaseous nebula might therefore be considered an extreme case of a Be star. If this is true, might there not be objects intermediate between Be stars and planetary nebulae? The answer is yes, but these objects are relatively few in number, indicating that in this intermediate condition equilibrium is seldom attained.

In normal Be stars the lines in emission are permitted laboratory lines (having high transition probabilities), while those most characteristic of gaseous nebulae are forbidden lines with very low transition probabilities. In intermediate spectra we might expect relative prominence of lines with intermediate transition probabilities, in other words, mildly forbidden lines. The lines $\lambda_{4363}$ [OIII] and $\lambda \lambda{ }_{4068 \text {, }}$ 4076 [SII] are examples. Stellar objects which appear to exemplify this intermediate class are: novae at certain phases, including RS Ophiuchi, the only star known to exhibit lines of the solar corona; $\eta$ Carinae, HD $45677, \mathrm{BD}+\mathrm{II}^{\circ} 4673$, RY Scuti, Il Puppis, HD 50r38; while among objects usually listed with planetary nebulae are IC 4997 and $\mathrm{BD}+30^{\circ} 3639$. These and a few fainter objects belonging to the same group might repay further spectroscopic and photometric study.

\section{Late Types}

Calcium emission has been found within the broad $\mathrm{H}$ and $\mathrm{K}$ lines of a number of G, $\mathrm{K}$ and $\mathrm{M}$ stars by Deslandres, and by Adams and Joy. In giant stars the bright portion is usually double, being divided by an absorption core whose intensity appears to be relatively high in the more luminous stars. The structure of the whole line is qualitatively similar to that of the hydrogen lines in early type spectra, Fig. Ia. In dwarf stars strong but single calcium emission within the broad dark lines is apparently characteristic. Bright $\mathrm{H}$ and $\mathrm{K}$, both single and double, are well known in the spectra of disturbed areas on the sun. Further study may well include intercomparison of solar and stellar phenomena.

A remarkable group of five or six $\mathrm{K}$ or $\mathrm{M}$ spectra having in emission forbidden lines of ionized iron deserves intensive study. Their potential interest is indicated by Christie's observation of a remarkable ultra-violet spectrum in VV Cephei.

Another small group of anomalous objects exhibits bright lines of ionized helium superposed on a spectrum with dark titanium oxide bands. The best known is $Z$ Andromedae, studied by $\mathrm{H}$. H. Plaskett and F. S. Hogg. Others are CI Cygni, AX Persei, RW Hydrae, and the nova-like variable T Coronae. The prominence of the nebular line $\lambda_{4363}$ suggests that these objects be considered in connection with the group previously referred to as intermediate between ordinary bright-line stars and planetary nebulae. The most amazing feature is the great intensity of $\lambda 4686$ of ionized helium. The upper atomic energy level for this line corresponds to an excitation of 50.8 volts, in addition to 24.5 volts required to ionize the atom. Since the second ionization potential of helium is 54.2 volts, only a little above the energy required for $\lambda 4686$, we might think that the emission of this line is part of a recombination process. If so, where do the doubly ionized helium atoms come from? A speculative explanation supposes the presence of radioactive atoms, possibly radioactive isotopes of relatively light elements, which supply the stripped helium nuclei in the form of alpha rays. $\lambda 4686$ is observable in the solar chromosphere, but because the intensity in integrated sunlight is negligible, an explanation 
based on local "hot spots" might be possible. The phenomenon, therefore, may or may not have the same cause as in the peculiar stars in whose spectra the line is conspicuous.

Let us now consider very briefly the fascinating problems presented by emission lines in the spectra of typical long-period variables. Bright hydrogen lines are common to variables of types $M, S$ and $N$, although in type $N$ the intensity is relatively low and some spectra may not have them at all.

In the laboratory, as well as in most astronomical sources, the bright lines in the Balmer series decrease in intensity regularly from $\mathrm{H} \alpha$ toward the violet, but in variables having titanium bands an extraordinary mutilation is regularly found. $\mathrm{H} \alpha$ and $\mathrm{H} \beta$ are weak or absent, $\mathrm{H} \gamma$ is well marked, while $\mathrm{H} \delta$ is often the strongest line in the spectrum. There is considerable evidence that these modifications are caused by the absorption of titanium oxide, although this explanation requires a. surprising inversion of the levels of emission and absorption. The distortion of the Balmer intensities varies with phase, being greatest when the lines first appear before maximum and tending to grow less toward maximum, and again toward minimum. This fact suggests that during their interval of appearance the lines are subject to the effect of band absorption in a decreasing degree. The striking weakness of bright $\mathrm{H} \epsilon$ relative to $\mathrm{H} \delta$ and $\mathrm{H} \zeta$ has long been considered due to the absorption of calcium in the broad $\mathrm{H}$ line.

The apparent splitting into two or three components of the $\mathrm{H} \gamma$ and $\mathrm{H} \delta$ lines (seen only with high dispersion) may, for a working hypothesis, be attributed also to absorption at higher levels, but strong doubt exists concerning this explanation. It is hoped before long to get a partial test from high-dispersion spectrograms of $\mathrm{Se}$ variables.

If, as is commonly believed, the conspicuous post-maximum lines $\lambda_{4202}$ and $\lambda 4308$ are the strong iron arc lines at these wave-lengths, then, since they have the same upper level, they should always be emitted with the same relative intensity. But observations show their relative intensity to vary, $\lambda 4308$ becoming relatively stronger with increasing interval after maximum light. Thus, if the identification is not in error, the lines must be affected by absorption in an overlying stratum. The absence of the lines $\lambda 1425 \mathrm{I}, 4272$ and 4326 in the same multiplet is puzzling and cannot well be ascribed to absorption. The explanation suggested by Thackeray and Merrill is similar to that advanced by Bowen to account for an incomplete multiplet of OIII in the spectra of nebulae, namely, the chance coincidence of a line from a very low level with a strong line of some other element. Excitation for the two iron lines may be furnished by an unobservable line of MgII.

As an Me variable fades toward minimum the hydrogen lines gradually disappear and $\lambda_{457} \mathrm{I}$, an extreme low-temperature line of magnesium, becomes conspicuous. Fainter bright lines at this pre-minimum phase include the numerous iron lines observed by King at the lowest working temperature of the electric furnace, the strongest being $\lambda 4376$. A valuable hypothesis by Wurm connects the emission of these and other low excitation lines with the energy made available by the formation of molecules in the star's atmosphere. The Balmer lines, requiring considerably higher excitation, are more difficult to explain.

A surprising phenomenon noted in four or five variables just before minimum may be characteristic. It is the appearance in emission of the forbidden lines of ionized iron. Can this be in some way a precursor of the new cycle? Shortly after minimum all bright lines disappear from the spectrum, $\lambda 457 \mathrm{I} \mathrm{Mg}$ being the last to go. 
A feature to which special attention should be drawn is the lack of symmetry in the behaviour of the bright lines with respect to the light-curve. Their aspect at a given magnitude before maximum differs markedly from that at the same magnitude after maximum. In general, no simple relationship between the behaviour of the bright lines and the photospheric temperature or any other single physical property is apparent. It seems rather that the brightening of the photosphere and the appearance of various emission lines are partially independent phenomena set going once each cycle by some recurring cause. The persistence of $\lambda 457 x$ after minimum suggests that the series of events so started may last longer than the period, one cycle partially overlapping another, just as on the sun a given series of spots may remain visible for two or three years after the next series begins.

A problem of long standing is that of the violet displacement of the bright lines with respect to the absorption spectrum. This unexplained shift is shown by all three types of red stars. The most numerous observations are of $\mathrm{Me}$ variables where the shift increases with the period from $0 \mathrm{~km}$. $/ \mathrm{sec}$. at Ioo days to $16 \mathrm{~km}$. $/ \mathrm{sec}$. at 400 days. Since the displacement occurs in different types of spectra and is shared by lines of hydrogen, iron, magnesium and one or two other elements, its cause must be a rather general one. A more specialized phenomenon is the additional shift of about $0.3 \mathrm{~A}$. toward the violet shown by $\mathrm{H} \alpha$ in $\mathrm{Me}$ spectra, for which a nearby band head of titanium oxide may be responsible. Displacements greater than normal are shown also by the bright lines of ionized calcium at $\lambda \lambda 8498,8542,8662$ in the infra-red. It is curious, by the way, that these lines should appear bright when no emission is seen at $\mathrm{H}$ and $\mathrm{K}$.

One definite bit of progress in the interpretation of complicated spectra has been made recently. The narrow conspicuous maxima in $\mathrm{N}$-type spectra have baffled investigators for a long time. They look like bright lines, but efforts to identify them were uniformly unsuccessful. Detailed comparisons with the spectrum of cyanogen by Merrill, Sanford and King now make it reasonably certain that many, and probably all, of the numerous unidentified maxima are only bits of continuous spectrum emerging through narrow interstices in the complex network of absorption lines.

The fact that the spectroscopic phenomena of long-period variables are very complex is at the same time a curse and a blessing: a curse because the observational data are difficult to obtain in sufficient refinement and because the interpretation is often obscure; a blessing because we have at hand a vast number of individual facts to suggest or to test physical hypotheses.

\section{LA COURONNE ET LES PROTUBERANCES EN DEHORS DES ÉCLIPSES}

\section{Par B. Lyot}

La rareté des éclipses totales de Soleil, leur faible durée et leur éloignement nous ont amené à rechercher une méthode qui permette d'étudier la couronne en tout temps.

Instrument. Notre méthode, appliquée depuis l'été I930, consiste à remplacer l'instrument d'observation par un coronographe spécialement étudié. Ce réfracteur a pour objectif une lentille simple de $20 \mathrm{~cm}$. d'ouverture, en verre très homogène, polie et nettoyée avec le plus grand soin. Un disque placé à son foyer arrête les rayons solaires directs, un système de lentilles et d'écrans placé après le foyer arrête 
la lumière diffractée par les bords de la première lentille ou réfléchie par le centre de ses surfaces et forme une image achromatique de la couronne.

Si l'on se sert d'un filtre rouge, la lumière diffusée par le coronographe, à 2 minutes du bord du Soleil, est du même ordre que celle de la couronne et environ 300 fois plus faible que la lumière diffusée par une bonne lunette de même ouverture.

En plaine, les poussières toujours présentes dans les basses couches de l'atmosphère produisent, autour du Soleil, une auréole de diffraction dont la brillance très variable dépasse généralement ioo fois celle de la couronne. A l'Observatoire du Pic du Midi, à l'altitude de 2877 mètres, le ciel est souvent d'une pureté parfaite et sa brillance, dans le rouge, devient inférieure à celle de la couronne.

Photographie de la couronne. Notre coronographe, installé dans cette station, permet alors de photographier, à travers un filtre rouge, la couronne intérieure jusqu'à $\eta^{\prime}$ du bord du Soleil.

Une série de clichés obtenus ainsi est projetée sur l'écran. Sur 5 images prises en I936, on voit des jets et des arches lumineuses dont la forme change en l'espace de 24 heures. Trois images à plus grande échelle prises le II mai I937 montrent la planète Mercure devant une protubérance puis devant la couronne, à 2' $37^{\prime \prime}$ du bord du Soleil. Six grands clichés pris en juillet r $_{93} 8$ permettent de constater les changements survenus dans la couronne pendant une même journée.

Spectre de la couronne. Un spectrographe à réseau plan, de $\mathrm{I} \mathrm{m}$. de foyer, était adapté au coronographe. Sa fente, en forme d'arc de cercle, permettait d'obtenir le spectre de la couronne à $40^{\prime \prime}$ des bords Est ou Ouest du Soleil, entre les latitudes + ou $-70^{\circ}$. Les spectres ont été pris en général dans le deuxième ordre, avec une dispersion de 7.5 A. par mm.; quelques-uns d'entre eux passent sur l'écran, ils sont sillonnés, dans le sens de la dispersion, par des bandes lumineuses dues au spectre continu des jets coronaux coupés par la fente. En plus des raies de Fraunhofer dues à la lumière solaire diffusée, on voit les raies de la couronne.

Dans l'ultra-violet, la diffusion atmosphérique est très intense et seule la forte raie 3388 apparaît.

Dans le vert, les conditions sont bien meilleures; en plus de la raie 5303 surexposée, les clichés montrent très nettement deux radiations, la raie 5 Ir 6 et une raie nouvelle de longueur d'onde $5694.42 \mathrm{~A}$., tandis que la raie 5536 n'apparaît sur aucune plaque.

Dans le rouge et l'infra-rouge, les clichés, poursuivis jusqu'à I2,000 A. en réduisant la dispersion, montrent les trois radiations connues: $6374 \cdot 5 \mathrm{I}, 670 \mathrm{I} \cdot 83$ et $789 \mathrm{I} \cdot 94 \mathrm{~A}$. et quatre raies nouvelles: $7059^{\circ} 62,8024 \cdot 2 \mathrm{I}$, I0 $74^{\circ} \cdot 80$ et ro797 95 A., les deux dernières étant parmi les plus fortes de tout le spectre coronal.

Les Ir raies coronales observées ont des intensités très variables d'un point à l'autre du bord du Soleil, leurs maxima occupent souvent des positions différentes et ne correspondent pas toujours à ceux du spectre continu. Des clichés de projections montrent, rassemblées sur une même plaque, les images de ces raies obtenues le même jour. Ils permettent de répartir les radiations qui possèdent la même distribution autour du Soleil en 3 groupes:

Le premier groupe comporte les raies $3388,5116,5303,6702,7059,8024$ et peut-être aussi ro747 et ro798.

Le deuxième groupe comporte seulement la nouvelle raie 5694 .

Le troisième groupe comporte les raies 6374 et 7892 .

Spectre des protubérances. En chacun des points où la fente de notre spectrographe coupait une protubérance brillante, il apparaît sur le cliché un spectre continu intense coupé par de nombreuses raies d'émission. Une série de plaques montre 
les raies de 1 hydrogène dans la série de Paschen, du sixième au trente-et-unième terme et dans la série de Balmer, jusqu'au trente-deuxième terme. A partir des limites de ces deux séries, le spectre continu de la protubérance se montre renforcé par celui de l'hydrogène.

Mouvements des protubérances. L'étude de ces mouvements a été faite pendant les années I935, I936 et I937, au moyen de films cinématographiques accélérés. Les vues, à l'échelle desquelles le Soleil mesure 50 à $75 \mathrm{~mm}$. de diamètre, ont été prises à la cadence de deux par minute, avec des temps de poses de $2 /$ Io à 3/ro de seconde, à travers notre filtre coloré isolant la radiation $\mathrm{H} \alpha$. Les films sont ensuite projetés à la vitesse normale; ils montrent les mouvements accélérés 600 fois.

Huit protubérances passent sur l'écran, leurs mouvements sont très variés. Une des plus intéressantes a été filmée au bord Ouest, le 8 août 1936 , de $8^{\text {h }}$ à $13^{\text {h }}$. Le sommet de cette formation montre des mouvements tourbillonnaires, ses divers points laissent échapper, vers le Sud, des filaments lumineux qui se précipitent dans une région très active de la chromosphère. Ce centre d'attraction, entouré par le dôme le plus brillant de la couronne intérieure, a été, à $I 7^{\mathrm{h}} 50$, après la fin du film, l'origine d'une éruption chromosphérique haute d'une centaine de secondes d'arc et visible avec une simple lunette.

Le meilleur film a été obtenu le I2 juin I937, de $8^{\text {h }} 30$ à $I 5^{\text {h }}$ Io, sur une protubérance située à l'Est et détachée du bord du Soleil. Pendant $4^{\mathrm{h}}$, celle-ci reste immobile tandis que la matière lumineuse s'en échappe dans diverses directions, étirée sous la forme de nombreux filaments très fins et incurvés qui aboutissent au Soleil. A $12^{\mathrm{h}} 4 \mathrm{o}$, une éruption chromosphérique jaillit sous la protubérance qu'elle atteint en quelques minutes. Toute la masse lumineuse, dont l'équilibre se trouve alors détruit, se disloque et s'écoule dans le Soleil en contournant un obstacle invisible qui entoure le lieu de l'éruption.

Conclusion. La comparaison des deux méthodes, avec et sans éclipse, met en lumière les faits suivants:

Pour photographier directement la couronne, seules les éclipses permettent d'atteindre les parties extérieures, la méthode sans éclipse ne montre que la couronne intérieure; en revanche elle permet seule d'étudier ses variations.

Pour étudier le spectre de la couronne:

Dans l'ultra-violet, le violet et le bleu, la diffusion atmosphérique est très forte et la méthode sans éclipse se montre très inférieure à l'autre.

Dans le vert, la diffusion est moins forte; on peut atténuer ses effets en augmentant la dispersion; les clichés plus nombreux et à plus grande échelle que ceux des éclipses permettent de discerner plus sûrement les radiations coronales faibles des agglomérations de grains; ils fournissent leurs longueurs d'ondes avec plus de précision.

Dans le rouge et surtout dans l'infra-rouge où les plaques photographiques sont peu sensibles, la méthode sans éclipse se montre très supérieure à l'autre.

Pour étudier les protubérances, la méthode sans éclipse permet seule d'attendre l'apparition d'objets très brillants qui présentent les spectres les plus riches et les plus intéressants. 